Pacific Journal of Mathematics

ON MASSEY PRODUCTS 


\title{
ON MASSEY PRODUCTS
}

\author{
EDWARD J. O'NEILL
}

\begin{abstract}
We present two examples: one showing the necessity of the condition that the two triple products must vanish simultaneously in order for the quadruple product to be defined; and one showing that the higher order products of a path connected, simply connected space are not completely determined by the differentials in the Eilenberg-Moore spectral sequence of its path-loop fibration.
\end{abstract}

1. Introduction. This paper is concerned with two examples concerning Massey products. Example I fills a gap in the literature since there are many references (see [2], [1], [3]) to the fact that the two triple products $\langle u, v, w\rangle$ and $\langle v, w, x\rangle$ must vanish simultaneously in order for the quadruple product $\langle u, v, w, x\rangle$ to be defined, yet there appears to be no example proving this. Example II gives a path connected, simply connected space which has higher order products which are not determined by the differentials in the Eilenberg-Moore spectral sequence of its path-loop fibration. This is to show that the higher products are a richer source of information about a space than the above-mentioned spectral sequence, and this should be contrasted with J. Peter May's result that matrix Massey products completely determine the differentials in the EilenbergMoore spectral sequence (see [4]).

2. Definitions. Because of different conventions in the literature used to define Massey products and to state what it means for the two triple products to vanish simultaneously, we present the following definitions:

Let $X$ be a topological space and $R$ a commutative ring with identity. $H^{*}(X ; R)$ will denote the singular cohomology ring and $C^{*}(X ; R)$ the singular cochain complex. (We could use in these definitions any cochain complex which has an associative product.) If $a \in H^{p}(X ; R)$ or $C^{p}(X ; R)$, we will write $\bar{a}=(-1)^{p} a$. We first define the triple product.

Definition 1. Let $u, v$, and $w$ be homogeneous entries from $H^{*}(X ; R)$ of degrees $p, q$, and $r$ respectively. Choose representative cocycles $u^{\prime}, v^{\prime}$, and $w^{\prime}$ for these classes respectively, and assume that $u v=0$ and $v w=0$. We may select cochains $\alpha^{12}$ and $\alpha^{23}$ such that

$$
\begin{aligned}
& \delta\left(a^{12}\right)=\overline{u^{\prime}} v^{\prime} \\
& \delta\left(a^{23}\right)=\overline{v^{\prime}} w^{\prime} .
\end{aligned}
$$


One can show that the cochain

$$
z^{\prime}=\overline{u^{\prime}} \alpha^{23}+\overline{\alpha^{12}} w^{\prime}
$$

is actually a cocycle of degree $p+q+r-1$.

We define the triple product $\langle u, v, w\rangle$ to be the collection of all cohomology classes $\left[z^{\prime}\right] \in H^{p+q+r-1}(X ; R)$ that we can obtain by the above procedure. This definition differs by the sign $(-1)$ from the definition in [3] and by the sign $(-1)^{q+1}$ from Massey's original definition in [6]. One can show that the indeterminacy of the triple product is

$$
u \cdot H^{q+r-1}(X ; R)+H^{p+q-1}(X ; R) \cdot w .
$$

In order to give the definition of the quadruple product $\langle u, v, w, x\rangle$, we must indicate what it means for the two triple products $\langle u, v, w\rangle$ and $\langle v, w, x\rangle$ to vanish simultaneously. Select representative cocycles $u^{\prime}, v^{\prime}, w^{\prime}, x^{\prime}$ respectively, for $u, v, w, x$ and assume that $u v=0$, $v w=0$, and $w x=0$. Then we may select cochains $a^{12}, a^{23}$, and $a^{34}$ such that

$$
\delta\left(\alpha^{12}\right)=\overline{u^{\prime}} v^{\prime}, \quad \delta\left(a^{23}\right)=\overline{v^{\prime}} w^{\prime}, \quad \delta\left(a^{34}\right)=\overline{w^{\prime}} x^{\prime}
$$

If we let

$$
y^{\prime}=\overline{u^{\prime}} a^{23}+\overline{a^{12}} w^{\prime} \quad \text { and } \quad z^{\prime}=\overline{v^{\prime}} \alpha^{34}+\overline{a^{23}} x^{\prime},
$$

these are cocycle representatives of $\langle u, v, w\rangle$ and $\langle v, w, x\rangle$ respectively. We say that $\langle u, v, w\rangle$ and $\langle v, w, x\rangle$ vanish simultaneously if it is possible to make the choices above so that both $y^{\prime}$ and $z^{\prime}$ are coboundaries. The purpose of Example $I$ is to show that it is possible for both $\langle u, v, w\rangle$ and $\langle v, w, x\rangle$ to vanish, but not to vanish simultaneously.

Definition 2. Let $u, v, w$ and $x$ be homogeneous entries from $H^{*}(X ; R)$ of degrees $p, q, r$ and $s$ respectively. Assume that

(1) $u v=0, v w=0, w x=0$

(2) $\langle u, v, w\rangle$ and $\langle v, w, x\rangle$ vanish simultaneously. Select representative cocycles $u^{\prime}, v^{\prime}, w^{\prime}$ and $x^{\prime}$ for $u, v, w$ and $x$ respectively, and because of condition (1) we may select cochains $a^{12}$, $a^{23}$, and $a^{34}$ such that

$$
\delta\left(\alpha^{12}\right)=\overline{u^{\prime}} v^{\prime}, \quad \delta\left(\alpha^{23}\right)=\overline{v^{\prime}} w^{\prime}, \quad \delta\left(a^{34}\right)=\overline{w^{\prime}} x^{\prime} .
$$

Making use of condition (2) we may assume that $a^{12}, a^{23}$, and $\alpha^{34}$ were selected so that there exist cochains $a^{13}$ and $a^{24}$ such that

$$
\delta\left(a^{13}\right)=\overline{u^{\prime}} a^{23}+\overline{a^{12}} w^{\prime} \text { and } \delta\left(a^{24}\right)=\overline{v^{\prime}} a^{34}+\overline{a^{23}} x^{\prime} .
$$


One can show that the cochain

$$
z^{\prime}=\overline{u^{\prime}} a^{24}+\overline{a^{12}} a^{34}+\overline{a^{13}} x^{\prime}
$$

is actually a cocycle of degree $p+q+r+s-2$.

We define the quadruple product $\langle u, v, w, x\rangle$ to be the collection of all cohomology classes $\left[z^{\prime}\right] \in H^{p+q+r+s-2}(X ; R)$ that we can obtain by the above procedure. This definition differs by the sign $(-1)$ from the definition in [3] and by the sign $(-1)^{p+r+1}$ from the definition in [2]. An element in the indeterminacy of the quadruple product can be written as an element of a marix triple product which we can write as

$$
\left\langle\left(u, z_{1}\right),\left(\begin{array}{cc}
v & z_{2} \\
0 & w
\end{array}\right),\left(\begin{array}{c}
z_{3} \\
x
\end{array}\right)\right\rangle
$$

where $z_{i} \in H^{*}(X ; R), 1 \leqq i \leqq 3$, by a result due to Kraines (see [3]).

3. ExAmple I. We will give an example of a topological space whose cohomology contains four classes $u_{1}, u_{2}, u_{3}$, and $u_{4}$ such that $\left\langle u_{1}, u_{2}, u_{3}\right\rangle$ and $\left\langle u_{2}, u_{3}, u_{4}\right\rangle$ are both defined and vanish, but do not vanish simultaneously. Thus in the cohomology of this space the quadruple product $\left\langle u_{1}, u_{2}, u_{3}, u_{4}\right\rangle$ is not defined, even though $\left\langle u_{1}, u_{2}, u_{3}\right\rangle=0$ and $\left\langle u_{2}, u_{3}, u_{4}\right\rangle=0$.

Let $X_{0}=\left(S_{1} V S_{2} V S_{3} V S_{4} V S_{5}\right) \bigcup_{f_{0}} e_{0}$ be the wedge of five oriented spheres $S_{i}$ of dimension $p_{i}, 1 \leqq i \leqq 5$, where $p_{5}=p_{2}+p_{3}-1$, with an oriented $\left(p_{1}+p_{2}+p_{3}-1\right)$-cell $e_{0}$ attached by the map $f_{0}$ which is a representative of the homotopy class $\left[i_{1},\left[i_{2}, i_{3}\right]\right]+\left[i_{1}, i_{5}\right]$, where $i_{j}$ denotes both the inclusion map $S_{j} \rightarrow S_{1} V S_{2} V S_{3} V S_{4} V S_{5}$ and its homotopy class, $1 \leqq j \leqq 5$. We will consider two spaces $X_{1}=X_{0} \cup_{f_{1}} e_{1}$ and $X_{2}=X_{0} \cup_{f_{2}} e_{2}$, where $e_{1}$ and $e_{2}$ are oriented $\left(p_{2}+p_{3}+p_{4}-1\right)$ cells, $f_{1}$ is a representative of the homotopy class $\left[i_{2},\left[i_{3}, i_{4}\right]\right]+\left[i_{5}, i_{4}\right]$, and $f_{2}$ is a representative of $\left[i_{2},\left[i_{3}, i_{4}\right]\right]-\left[i_{5}, i_{4}\right]$.

In $H^{*}\left(X_{1} ; \boldsymbol{Z}\right)$ and $H^{*}\left(X_{2} ; \boldsymbol{Z}\right)$ we will let $u_{i}$ denote the cohomology class represented by the cocycle which assigns +1 to the cell $S_{i}$ and 0 elsewhere, $1 \leqq i \leqq 5$, and $z_{i}$ denote the class represented by the cocycle which assigns +1 to the cell $e_{i}$ and 0 elsewhere, $0 \leqq i \leqq 2$. Then using Lemma 7 in [6], in $H^{*}\left(X_{1} ; Z\right)$ we have

$$
\left\langle u_{1}, u_{2}, u_{3}\right\rangle=(-1)^{p_{1}+p_{2}+1} z_{0}=(-1)^{p_{1}+p_{2}+1} u_{1} u_{5}
$$

and

$$
\left\langle u_{2}, u_{3}, u_{4}\right\rangle=(-1)^{p_{2}+p_{3}+1} z_{1}=(-1)^{p_{2}+p_{3}+1} u_{5} u_{4} \text {. }
$$

Similarly in $H^{*}\left(X_{2} ; \boldsymbol{Z}\right)$ we have $\left\langle u_{1}, u_{2}, u_{3}\right\rangle=(-1)^{p_{1}+p_{2}+1} z_{0}=(-1)^{p_{1}+p_{2}+1} u_{1} u_{5}$ and $\left\langle u_{2}, u_{3}, u_{4}\right\rangle=(-1)^{p_{2}+p_{3}+1} z_{2}=(-1)^{p_{2}+p_{3}} u_{5} u_{4}$. Since $H^{*}\left(X_{i}\right) \cdot u_{3}=0$ and $u_{2} \cdot H^{*}\left(X_{i}\right)=0$, the indeterminacy of $\left\langle u_{1}, u_{2}, u_{3}\right\rangle$ is $u_{1} \cdot H^{*}\left(X_{i}\right)$ 
and the indeterminacy of $\left\langle u_{2}, u_{3}, u_{4}\right\rangle$ is $H^{*}\left(X_{i}\right) \cdot u_{4}, i=1,2$. Thus for both $X_{1}$ and $X_{2},\left\langle u_{1}, u_{2}, u_{3}\right\rangle$ and $\left\langle u_{2}, u_{3}, u_{4}\right\rangle$ both vanish. Depending on the parity of $p_{2}$, either $X_{1}$ or $X_{2}$ will be the space required.

As in [2] on p. 148, we can define in our situation an operation denoted by $\left(\left\langle u_{1}, u_{2}, u_{3}\right\rangle,\left\langle u_{2}, u_{3}, u_{4}\right\rangle\right)$ which consists of all ordered pairs $\left(\overline{u_{1}^{\prime}} a^{23}+\overline{a^{12}} u_{3}^{\prime}, \overline{u_{2}^{\prime}} a^{34}+\overline{a^{23}} u_{4}^{\prime}\right)$ that one can obtain, where previous notation has been used here. One can see that in our situation the operation $\left(\left\langle u_{1}, u_{2}, u_{3}\right\rangle,\left\langle u_{2}, u_{3}, u_{4}\right\rangle\right)$ is a coset of $H^{p_{1}+p_{2}+p_{3}-1}\left(X_{i}\right) \times$ $H^{p_{2}+p_{3}+p_{4}-1}\left(X_{i}\right)$ modulo the subgroup Image $(\Phi)$, where $\Phi: H^{p_{2}+p_{3}-1}\left(X_{i}\right) \rightarrow$ $H^{p_{1}+p_{2}+p_{3}-1}\left(X_{i}\right) \times H^{p_{2}+p_{3}+p_{4}-1}\left(X_{i}\right)$ is defined by $\Phi(z)=\left(\bar{u}_{1} z, \bar{z} u_{4}\right)$. For our example we desire $\left(\left\langle u_{1}, u_{2}, u_{3}\right\rangle,\left\langle u_{2}, u_{3}, u_{4}\right\rangle\right) \notin$ Image $(\Phi)$.

But if $p_{2}$ is even, then in $H^{*}\left(X_{1}\right)$ we have

$$
\begin{aligned}
\left(\left\langle u_{1}, u_{2}, u_{3}\right\rangle,\left\langle u_{2}, u_{3}, u_{4}\right\rangle\right) & =\left((-1)^{p_{1}+p_{2}+1} u_{1} u_{5},(-1)^{p_{2}+p_{3}+1} u_{5} u_{4}\right) \\
& =\left(-\bar{u}_{1} u_{5}, \bar{u}_{5} u_{4}\right) \notin \text { Image }(\Phi) .
\end{aligned}
$$

And if $p_{2}$ is odd, then in $H^{*}\left(X_{2}\right)$ we have

$$
\begin{aligned}
\left(\left\langle u_{1}, u_{2}, u_{3}\right\rangle,\left\langle u_{2}, u_{3}, u_{4}\right\rangle\right) & =\left((-1)^{p_{1}+p_{2}+1} u_{1} u_{5},(-1)^{p_{2}+p_{3}} u_{5} u_{4}\right) \\
& =\left(\bar{u}_{1} u_{5},-\bar{u}_{5} u_{4}\right) \notin \text { Image }(\Phi), \text { as desired. }
\end{aligned}
$$

Note also that if $p_{2}$ is even, in $H^{*}\left(X_{2}\right)$, or if $p_{2}$ is odd, in $H^{*}\left(X_{1}\right)$, we have examples of spaces where the quadruple product is defined, but is not strictly defined in the sense of May (see [3], p. 538).

4. ExAMPLE II. The differentials in the Eilenberg-Moore spectral sequence associated with the path-loop fibration of a path connected simply connected space are completely determined by higher order Massey products (see [3]). This might lead one to conjecture that the differentials in the spectral sequence contain all the information about the higher order products that our definition contains. But to see that this is not the case, we will consider the EilenbergMoore spectral sequence of the path-loop fibration of the following space.

Let $X_{0}=S_{1} V S_{2} V S_{3} V S_{4}$ be the wedge of four oriented spheres, where $\operatorname{dim} S_{i}=p_{i}>1, p_{4}=p_{1}+p_{3}-1$, and we let $r=p_{1}+p_{2}+p_{3}-2$. Let $i_{j}$ denote the homotopy class of the inclusion $S_{j} \rightarrow X_{0}$ and let $f$ be a map from an oriented $r$-sphere into $X_{0}$ which represents $\left[i_{1}\right.$, $\left.\left[i_{2}, i_{3}\right]\right]-\left[i_{2}, i_{4}\right]$. If $e$ is an oriented $(r+1)$-cell, we may attach $e$ to $X_{0}$ via the map $f$ and call the resulting space $X_{1}$. In $H^{*}\left(X_{1} ; Z\right)$ let $u_{i}$ be the cohomology class which is represented by the cocycle which assigns +1 to $S_{i}$ and 0 elsewhere, and let $z$ be the cohomology class represented by the cocycle which assigns +1 to $e$ and 0 elsewhere. 
By using Lemma 7 in [6], we can easily show that the triple product $\left\langle u_{1}, u_{2}, u_{3}\right\rangle=(-1)^{p_{1}+p_{2}} u_{2} u_{4}=(-1)^{p_{1}+p_{2}+1} z$ and thus is nontrivial; however in the Eilenberg-Moore spectral sequence associated with the path-loop fibration of $X_{1}$, this triple product, being decomposable, would be trivial.

\section{REFERENCES}

1. D. Kraines, Massey higher products, Trans. Amer. Math. Soc., 124 (1966), 431-449. 2. W. S. Massey, Some higher order cohomology operations, Symposium International de Topologia Algebraica, Mexico City, (1958), 145-154

3. J. P. May, Matric massey products, J. Algebra, 12 (1969), 533-568.

4. - The cohomology of principle bundles, homogeneous spaces, and two-stage Postnikov-systems, Bull. Amer. Math. Soc., 74 (1968), 334-339.

5. E. J. O'Neill, Higher Order Cohomology Products and Links, Ph. D. Dissertation, Yale University, 1976.

6. H. Uehara and W. S. Massey, The Jacobi identity for Whitehead products, Algebraic Geometry and Topology, a Symposium in honor of S. Lefschetz, Princeton University Press, 1957, 361-377.

Received July 28, 1977. These results are taken from a dissertation [5] written under the direction of William S. Massey. The author wishes to express his gratitude to Professor Massey for his guidance, encouragement and suggestions during its preparation.

FAIRFIELD UNIVERSITY

FAIRFIELD, CT 06430 



\section{PACIFIC JOURNAL OF MATHEMATICS}

\section{EDITORS}

RICHARD ARENS (Managing Editor)

University of California

Los Angeles, California 90024

C. W. CURTIS

University of Oregon

Eugene, OR 97403

C. C. MOORE

University of California

Berkeley, CA 94720

\section{J. DUGUNDJI}

Department of Mathematics University of Southern California Los Angeles, California 90007

R. Finn aNd J. Milgram Stanford University Stanford, California 94305

\section{ASSOCIATE EDITORS}

E. F. BeCKenbaCH

B. H. NeumanN

F. WOLF

K. YosHIDA

\section{SUPPORTING INSTITUTIONS}

UNIVERSITY OF BRITISH COLUMBIA CALIFORNIA INSTITUTE OF TECHNOLOGY UNIVERSITY OF CALIFORNIA MONTANA STATE UNIVERSITY UNIVERSITY OF NEVADA, RENO NEW MEXICO STATE UNIVERSITY OREGON STATE UNIVERSITY UNIVERSITY OF OREGON
UNIVERSITY OF SOUTHERN CALIFORNIA STANFORD UNIVERSITY UNIVERSITY OF HAWAII UNIVERSITY OF TOKYO UNIVERSITY OF UTAH WASHINGTON STATE UNIVERSITY UNIVERSITY OF WASHINGTON 


\section{Pacific Journal of Mathematics}

\section{Vol. 76, No. $1 \quad$ November, 1978}

Ata Nuri Al-Hussaini, Potential operators and equimeasurability ......... 1

Tim Anderson and Erwin Kleinfeld, Semisimple nil algebras of type $\delta . \ldots .99$

Stephen LaVern Campbell, Linear operators for which $T^{*} T$ and $T+T^{*}$

commute. III ......................................

Robert Jay Daverman, Special approximations to embeddings of codimension one spheres...............................

Donald M. Davis, Connective coverings of $\mathrm{BO}$ and immersions of projective

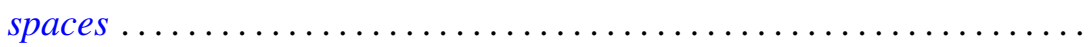

V. L. (Vagn Lundsgaard) Hansen, The homotopy type of the space of maps of

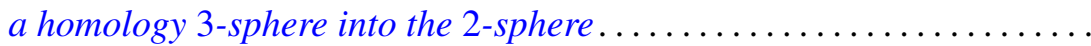

James Victor Herod, A product integral representation for the generalized

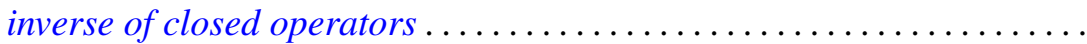

A. A. Iskander, Definability in the lattice of ring varieties ..............

Russell Allan Johnson, Existence of a strong lifting commuting with a

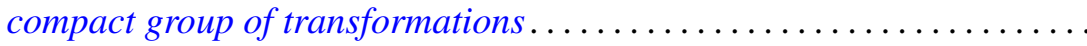

Heikki J. K. Junnila, Neighbornets...................... 83

Klaus Kalb, On the expansion in joint generalized eigenvectors . ......... 109

F. J. Martinelli, Construction of generalized normal numbers . . . . . . . . . 117

Edward O'Neill, On Massey products ....................... 123

Vern Ival Paulsen, Continuous canonical forms for matrices under unitary

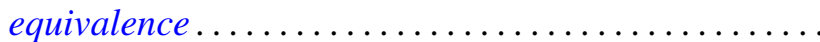

Justin Peters and Terje Sund, Automorphisms of locally compact groups . . . 143

Duane Randall, Tangent frame fields on spin manifolds . . . .

Jeffrey Brian Remmel, Realizing partial orderings by classes of co-simple sets . . . .

J. Hyam Rubinstein, One-sided Heegaard splittings of 3-manifolds ...

Donald Charles Rung, Meier type theorems for general boundary approach

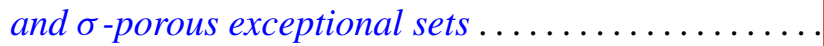

Ryōtarō Satō, Positive operators and the ergodic theorem

Ira H. Shavel, A class of algebraic surfaces of general type constructed from

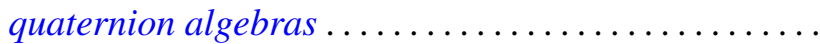

Patrick F. Smith, Decomposing modules into projectives and injectives ....

Sergio Eduardo Zarantonello, The sheaf of outer functions in the polydisc... 